\title{
Iterative State Estimation
}

\author{
Thomas J. Riedl and Andrew C. Singer \\ University of Illinois at Urbana-Champaign \\ Urbana, IL 61801, USA \\ Email: tried12@illinois.edu
}

\begin{abstract}
Iterative solvers allow for a trade-off between speed and accuracy. We propose an iterative method for the estimation of the internal states of a given discrete-time linear state-space model from a series of noisy measurements. In particular we identify the MAP estimate of those states as being the solution of a sparse system of linear equations and derive an iterative solver based on the conjugate gradient method. We derive convergence results to quantify the trade-off between speed and accuracy and finally apply the method to channel estimation where it is shown to outperform Kalman smoothing complexity-wise.

Index Terms-state space systems, conjugate gradient method, state estimation, Kalman smoothing.
\end{abstract}

\section{INTRODUCTION}

Many problems in signal processing can be cast into the framework of state estimation, in which we have state variables $\boldsymbol{h}$ whose values are not directly accessible and variables $\boldsymbol{y}$ whose values are available and are statistically dependent on $\boldsymbol{h}$. In particular it is assumed that the conditional distribution $p(\boldsymbol{y} \mid \boldsymbol{h})$ is given. The variables $\boldsymbol{y}$ are also referred to as observations or measurements in this context. Due to the statistical dependence between the states $\boldsymbol{h}$ and the observations $\boldsymbol{y}$, the observations can be used to infer an estimate $\hat{h}$ of the states. The estimate $\hat{\boldsymbol{h}}$ given $\boldsymbol{y}$ could be randomized, but in this paper we assume the estimate $\hat{\boldsymbol{h}}$ to be a deterministic function of $\boldsymbol{y}$. This setup is schematically depicted in Figure I.

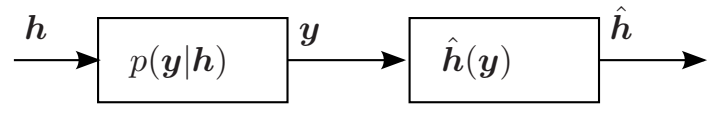

Figure 1. State Estimation

In discrete-time linear state-space models the evolution of the state vectors $\boldsymbol{h}[i]$ in $\boldsymbol{h}$ for $i=0, \ldots, M-1$ is described by a Markov chain built on linear operators perturbed by Gaussian noise. The statistical relationship $p(\boldsymbol{y} \mid \boldsymbol{h})$ between the state variables $\boldsymbol{h}$ and the observations $\boldsymbol{y}$ is assumed to be Gaussian with mean linear in $\boldsymbol{h}$. Because of its great flexibility and the existence of computational efficient methods for the inference of its states this model is very popular and has been applied to many signal processing and communications problems (see [1], [2] for example).

The exact MAP estimate of the states of such a discrete-time linear state-space model can be obtained by use of Kalman

This work was supported in part by the department of the Navy, Office of Naval Research, under grants ONR MURI N00014-07-1-0738 and ONR N00014-07-1-0311. smoothing. The complexity of the standard implementation of Kalman smoothing is $\mathcal{O}\left(L^{3} M\right)$ [3], where $L$ denotes the dimensionality of $\boldsymbol{h}[i]$. In many problems, however, the state variables $\boldsymbol{h}[i]$ are very high-dimensional and Kalman smoothing is therefore intractable.

In this paper we propose an iterative solver whose iterates are vectors that approach the exact MAP estimate. The main contribution is a convergence result that upper bounds the number of iterations necessary to achieve an iterate $\epsilon$ close to the exact MAP estimate. This result applies to a broad class of discrete-time linear state-space models and given a model from this class we will show that the computational complexity required to obtain an iterate arbitrary close to the exact MAP estimate is $\mathcal{O}\left(L^{5 / 2} M\right)$.

We finally apply the method to channel estimation where it is shown to outperform Kalman smoothing complexity-wise.

\section{Notation And System Definition}

The discrete-time linear state-space model considered in this paper has the following form:

$$
\begin{aligned}
\boldsymbol{h}[i+1] & =\boldsymbol{T} \boldsymbol{h}[i]+\boldsymbol{w}[i] \\
\boldsymbol{y}[i] & =\boldsymbol{X}[i] \boldsymbol{h}[i]+\boldsymbol{n}[i] \\
i & =0, \ldots, M-1
\end{aligned}
$$

where $\boldsymbol{h}[i] \in \mathbb{C}^{L}, \boldsymbol{y}[i] \in \mathbb{C}^{P}$, the transition matrix $\boldsymbol{T} \in \mathbb{C}^{L \times L}$ is assumed to be hermitian and full rank and $\boldsymbol{h}[0], \boldsymbol{w}[i]$ and $\boldsymbol{n}[i]$ are assumed to be independent and Gaussian distributed with zero mean and covariance matrix $\boldsymbol{\Sigma}_{0}, \boldsymbol{\Sigma}_{w}$ and $\boldsymbol{\Sigma}_{n}$, respectively. Furthermore, in this paper we will assume that the matrices $\boldsymbol{T}, \boldsymbol{\Sigma}_{0}$ and $\boldsymbol{\Sigma}_{w}$ commute. For notational convenience we concatenate the vectors $\boldsymbol{y}[i] \forall i$ and $\boldsymbol{h}[i] \forall i$ vertically and in increasing order and denote the results by $\boldsymbol{y}$ and $\boldsymbol{h}$, respectively. The dimension of these vectors is $P * M$ and $L * M$, respectively. The inverse covariance matrix $\Sigma_{h}^{-1}$ of the zero-mean Gaussian distribution $p(\boldsymbol{h})$ then exhibits the following block tridiagonal form:

$$
\boldsymbol{\Sigma}_{h}^{-1}=\left[\begin{array}{cccc}
\boldsymbol{\Sigma}_{0}^{-1}+\boldsymbol{T}^{2} \boldsymbol{\Sigma}_{w}^{-1} & -\boldsymbol{T} \boldsymbol{\Sigma}_{w}^{-1} & & \\
-\boldsymbol{T} \boldsymbol{\Sigma}_{w}^{-1} & \boldsymbol{T}^{2} \boldsymbol{\Sigma}_{w}^{-1}+\boldsymbol{\Sigma}_{w}^{-1} & \ddots & \\
\ddots & & \ddots & \\
& & & \\
& & & \boldsymbol{\Sigma}_{w}^{-1}
\end{array}\right]
$$


The conditional distribution $p(\boldsymbol{y} \mid \boldsymbol{h})$ factors

$$
p(\boldsymbol{y} \mid \boldsymbol{h})=\prod_{i} p(\boldsymbol{y}[i] \mid \boldsymbol{h}[i])
$$

and the distributions $p(\boldsymbol{y}[i] \mid \boldsymbol{h}[i])$ are Gaussian with mean $\boldsymbol{X}[i] \boldsymbol{h}[i]$ and covariance matrix $\boldsymbol{\Sigma}_{n}$.

\section{iII. State Estimation}

\section{A. Problem setup}

The state estimation task is based on the following MAP estimation approach:

$$
\begin{aligned}
\hat{\boldsymbol{h}} & =\underset{\boldsymbol{h}}{\operatorname{argmax}} p(\boldsymbol{h} \mid \boldsymbol{y}) \\
& =\underset{\boldsymbol{h}}{\operatorname{argmax}} p(\boldsymbol{y} \mid \boldsymbol{h}) p(\boldsymbol{h})
\end{aligned}
$$

Note that this MAP estimate coincides with the MMSE estimate of $\boldsymbol{h}$ as $\boldsymbol{h}$ and $\boldsymbol{y}$ are jointly Gaussian.

\section{B. Solution}

Summarizing all the quadratic terms and summarizing all the linear terms in the exponent of $p(\boldsymbol{y} \mid \boldsymbol{h}) p(\boldsymbol{h})$ shows that

$$
p(\boldsymbol{y} \mid \boldsymbol{h}) p(\boldsymbol{h}) \sim \exp \left(-\boldsymbol{h}^{H}\left(\boldsymbol{\Sigma}_{h}+\boldsymbol{X}\right) \boldsymbol{h}+\boldsymbol{b}^{H} \boldsymbol{h}+\boldsymbol{h}^{H} \boldsymbol{b}\right)
$$

where

$$
\begin{gathered}
\boldsymbol{X}=\left[\begin{array}{ccc}
\boldsymbol{X}[0]^{H} \boldsymbol{\Sigma}_{n}^{-1} \boldsymbol{X}[0] & \\
& \boldsymbol{X}[1]^{H} \boldsymbol{\Sigma}_{n}^{-1} \boldsymbol{X}[1] & \\
& \ddots
\end{array}\right] \\
\boldsymbol{b}=\left[\begin{array}{c}
\boldsymbol{X}[0]^{H} \boldsymbol{\Sigma}_{n}^{-1} \boldsymbol{y}[0] \\
\boldsymbol{X}[1]^{H} \boldsymbol{\Sigma}_{n}^{-1} \boldsymbol{y}[1] \\
\vdots
\end{array}\right]
\end{gathered}
$$

In other words, $p(\boldsymbol{y} \mid \boldsymbol{h}) p(\boldsymbol{h})$ is proportional to a Gaussian distribution with inverse covariance matrix $\boldsymbol{A}=\left(\boldsymbol{\Sigma}_{h}+\boldsymbol{X}\right)$ and mean $\boldsymbol{m}=\boldsymbol{A}^{-1} \boldsymbol{b}$. Gaussian densities assume their maximum at their mean and therefore the optimization problem above amounts to calculating the mean vector $\boldsymbol{m}$, which in turn is the solution of the sparse linear system $\boldsymbol{A} \boldsymbol{m}=\boldsymbol{b}$. Gaussian elimination is theoretically able to solve any full rank linear system and hence would in principle work for the above linear system as well, but its cubic complexity becomes prohibitive for problems of high dimensionality. Exploiting the structure of our positive definite system matrix $\boldsymbol{A}$, however, allows us to construct a far more efficient solver as we shall see in the following.

\section{The Conjugate Gradient Method}

The solution method we are going to propose is based on the conjugate gradient method which we shall introduce briefly in this section. Starting from an initial guess $\boldsymbol{m}^{(0)}$ the conjugate gradient method successively increases the order $k$ of the Krylov space

$$
U_{k}=\operatorname{span}\left\{\boldsymbol{r}^{(0)}, \boldsymbol{A} \boldsymbol{r}^{(0)}, \ldots, \boldsymbol{A}^{k-1} \boldsymbol{r}^{(0)}\right\},
$$

where $\boldsymbol{r}^{(0)}=\boldsymbol{b}-\boldsymbol{A} \boldsymbol{m}^{(0)}$, and determines the iterate $\boldsymbol{m}^{(k)}$ as the solution of

$$
\min _{\boldsymbol{m}^{(k)} \in \boldsymbol{m}^{(0)}+U_{k}}\left\|\boldsymbol{m}^{(k)}-\boldsymbol{m}\right\|_{\boldsymbol{A}}
$$

where the norm $\|\cdot\|_{A}$ is induced by the inner product

$$
(\boldsymbol{x}, \boldsymbol{y})_{\boldsymbol{A}}=(\boldsymbol{A} \boldsymbol{x}, \boldsymbol{y})=\boldsymbol{x}^{H} \boldsymbol{A} \boldsymbol{y}
$$

By the Cayley-Hamilton theorem there is a polynomial $p$ of degree $N-1$ such that $\boldsymbol{A}^{-1}=p(\boldsymbol{A})$. So

$\boldsymbol{m}-\boldsymbol{m}^{(0)}=\boldsymbol{A}^{-1}\left(\boldsymbol{b}-\boldsymbol{A} \boldsymbol{m}^{(0)}\right)=\boldsymbol{A}^{-1} \boldsymbol{r}^{(0)}=p(\boldsymbol{A}) \boldsymbol{r}^{(0)} \in U_{n}$,

$\boldsymbol{m} \in \boldsymbol{m}^{(0)}+U_{n}$ and the conjugate gradient method obtains the solution $\boldsymbol{m}$ in at most $N$ steps. The complexity of each conjugate gradient step is dominated by the complexity of the matrix vector product $\boldsymbol{A} \boldsymbol{p}^{(k)}$ for some $\boldsymbol{p}^{(k)} \in \mathbb{C}^{N}$ [4]. In our case $\boldsymbol{A}$ is block tridiagonal and hence this multiplication has complexity at most $\mathcal{O}\left(L^{2} M\right)$. In fact in some cases the structure of the blocks of $\boldsymbol{\Sigma}_{h}^{-1}$ and $\boldsymbol{X}$ allow for a per-step complexity linear in $L$. For example when the matrices $\Sigma_{0}$ and $\Sigma_{w}$ are diagonal, $\boldsymbol{T}$ has small bandwidth and the blocks in $\boldsymbol{X}$ are rank one as in the example investigated in section VI. For the determination of the overall complexity, the above complexity needs of course be multiplied with the number of necessary iterations. As mentioned it takes in general up to $N$ steps to reach the solution $\boldsymbol{m}$. Often, however, convergence is achieved in far less than $N$ steps with the speed of the convergence depending solely on the spectrum of $\boldsymbol{A}$ [4]. This motivates the convergence analysis of the next section.

\section{Convergence Analysis}

Determining the spectrum of $\boldsymbol{A}$ analytically is hard, but in our case we can at least give an upper bound on the condition number $\kappa_{2}(\boldsymbol{A})$ of $\boldsymbol{A}$. The following theorem can then be used to give an upper bound on the convergence rate [4].

Theorem 1. Suppose $\boldsymbol{A} \in \mathbb{C}^{N \times N}$ is positive definite. If the conjugate gradient method produces iterates $\boldsymbol{m}^{(k)}$ and $\kappa=$ $\kappa_{2}(\boldsymbol{A})$ then

$$
\left\|\boldsymbol{m}-\boldsymbol{m}^{(k)}\right\|_{\boldsymbol{A}} \leq 2\left\|\boldsymbol{m}-\boldsymbol{m}^{(0)}\right\|_{\boldsymbol{A}}\left(\frac{\sqrt{\kappa}-1}{\sqrt{\kappa}+1}\right)^{k}
$$

This statement can be used to give an upper bound on the number of iterations necessary to achieve a given accuracy [5].

\section{Corollary 1.}

$$
k \leq \frac{-\ln \left(\frac{1}{2} \frac{e}{e_{0}}\right)}{\ln \left(\frac{\sqrt{\kappa}+1}{\sqrt{\kappa}-1}\right)} \leq-\ln \left(\frac{1}{2} \frac{e}{e_{0}}\right) \frac{1}{2} \sqrt{\kappa}
$$

In the remainder of this section is dedicated to the development of an upper bound on $\kappa$. We start with an theorem bounding the extramal eigenvalues of the inverse covariance matrix $\boldsymbol{\Sigma}_{h}^{-1}$.

Theorem 2. Suppose the matrices $\boldsymbol{T}, \boldsymbol{\Sigma}_{0}$ and $\boldsymbol{\Sigma}_{w}$ commute, the spectral radius $\rho(\boldsymbol{T}) \leq 1$ then the following inequalities 
regarding the extramal eigenvalues of the inverse covariance matrix $\Sigma_{h}^{-1}$ as defined in (4) hold ${ }^{1}$

$$
\begin{aligned}
\lambda_{\min }\left(\boldsymbol{\Sigma}_{h}^{-1}\right) \geq & \min _{i} \min \left\{\lambda_{0, i}^{-2} \lambda_{w, i},\left(1-\left|\lambda_{T, i}\right|\right)^{2} \lambda_{w, i}^{-1}\right\} \\
\equiv & \underline{\lambda}_{\min }\left(\boldsymbol{\Sigma}_{h}^{-1}\right) \\
\lambda_{\max }\left(\boldsymbol{\Sigma}_{h}^{-1}\right) \leq & \max _{i}\left\{\left(1+\left|\lambda_{T, i}\right|\right)^{2} \lambda_{w, i}^{-1}+\max \left(\lambda _ { w , i } ^ { - 1 } \left(\left|\lambda_{T, i}\right|-\right.\right.\right. \\
& \left.\left.\left.\quad-\lambda_{T, i}^{2}\right),-\lambda_{w, i}^{-1}+\lambda_{0, i}^{-1}+\left|\lambda_{T, i}\right| \lambda_{w, i}^{-1}\right)\right\} \\
\equiv & \bar{\lambda}_{\max }\left(\boldsymbol{\Sigma}_{h}^{-1}\right)
\end{aligned}
$$

where $\lambda_{T, i}, \lambda_{0, i}$ and $\lambda_{w, i}$ denote the eigenvalues of $\boldsymbol{T}, \boldsymbol{\Sigma}_{0}$ and $\boldsymbol{\Sigma}_{w}$, respectively, corresponding to the $i$-th joint eigenvector.

Remarkably, those bounds do not depend on $M$. The proof of theorem 2 is inspired by the eigenvalue analysis of tridiagonal matrices as arising from the discrete Poisson equation [6]. The complete proof can be found in [7].

The matrix $\boldsymbol{X}$ is positive semidefinite and so we arrive at the following trivial lower and upper bounds:

$$
\begin{aligned}
\lambda_{\min }(\boldsymbol{A}) & \geq \underline{\lambda}_{\min }\left(\boldsymbol{\Sigma}_{h}^{-1}\right) \\
\lambda_{\max }(\boldsymbol{A}) & \leq \bar{\lambda}_{\max }\left(\boldsymbol{\Sigma}_{h}^{-1}\right)+\lambda_{\max }(\boldsymbol{X})
\end{aligned}
$$

The above results now allow us to make an interesting observation. Assume that there are non-zero lower and upper bounds $c_{1}$ and $c_{2}$ such that

$$
c_{1} \leq \underline{\lambda}_{\min }\left(\boldsymbol{\Sigma}_{h}^{-1}\right) \leq \bar{\lambda}_{\max }\left(\boldsymbol{\Sigma}_{h}^{-1}\right) \leq c_{2} \forall L
$$

and further assume that there is a constant $c_{3}$ such that $\lambda_{\max }\left(\boldsymbol{X}[i]^{H} \boldsymbol{\Sigma}_{n}^{-1} \boldsymbol{X}[i]\right) \leq c_{3} L \forall L$ and $\forall i$. Then

$$
\kappa(\boldsymbol{A}) \leq \frac{c_{2}+c_{3} L}{c_{1}}
$$

and the overall complexity of the conjugate gradient algorithm is $\mathcal{O}\left(L^{5 / 2} M\right)$. Note that the complexity of a possible Kalman smoothing solution to the same problem would be $\mathcal{O}\left(L^{3} M\right)$ which is higher. We will see a relevant class of problems that meets the above assumptions in the next section.

\section{Vi. Application and Simulation Results}

We will now illustrate the performance of the scheme presented above by an example application in communications. If we choose $\boldsymbol{T}$ to be $\alpha \mathbf{I}$ and assume both the noise $\boldsymbol{w}[i]$ and the initial state $\boldsymbol{h}[0]$ to be vectors of i.i.d. Gaussians with variance $\sigma_{w}^{2}$ and $\sigma_{0}^{2}$, respectively, the state evolution equation (1) represents a first order autoregressive model for a frequency-selective Rayleigh fading channel [8], [9]. The vector $\boldsymbol{h}[i]$ comprises $L$ independent Rayleigh processes and the matrix $\boldsymbol{X}[i]$ in the output equation (2) becomes the data vector $\boldsymbol{x}[i]^{T}$ in case of a simple Single-Input and Single-Output system. For simplicity, the symbols in $\boldsymbol{x}[i]^{T}$ are assumed to be phase modulated and we require the average symbol energy to be normalized to one, i.e., $\left|\boldsymbol{x}[i]_{j}\right|^{2}=1$. Note, that in this case the inverse noise variance $\sigma_{n}^{-2}$ coincides with the Signal-to-Noise ratio. For the actual simulations, we set

\footnotetext{
${ }^{1}$ We write $M>N$ if $M-N>0$; i.e., $M-N$ is positive definite. Similarly for ' $\geq$ '.
}

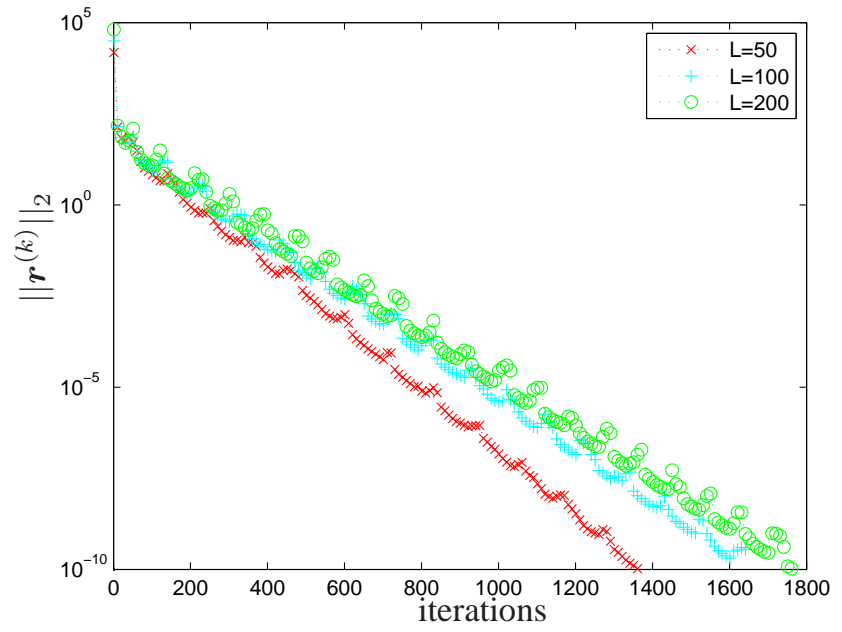

Figure 2. Residual norm $\left\|\boldsymbol{r}^{(k)}\right\|_{2}$ over the number of iterations for different choices of $L$ and $M=1000$

$\sigma_{0}^{2}=\sigma_{w}^{2}$ and obtain for a normalized maximum doppler of $f_{m}=0.05$ (this is the same value as used for performance evaluation in [8]) the values $\alpha=0.9755$ and $\sigma_{w}^{2}=0.0484$. With this configuration and $\sigma_{n}^{2}=0.1$

$$
\kappa(\boldsymbol{A}) \leq \frac{4 \sigma_{w}^{-2} \alpha+\sigma_{0}^{-2}+L \sigma_{n}^{-2}}{\sigma_{w}^{-2}(1-\alpha)^{2}} \approx 8151+806 L
$$

and the overall complexity of the algorithm is $\mathcal{O}\left(L^{3 / 2} M\right)$. Figure 2 shows a plot of the residual norm $\left\|\boldsymbol{r}^{(k)}\right\|_{2}$ over the number of iterations for different choices of $L$. We recognize that the proposed method exhibits linear convergence and that the number of iterations necessary for a given accuracy scales sublinearly with $L$.

\section{REFERENCES}

[1] D. Barber, "Efficient Kalman Smoothing for Harmonic State-Space Models," Tech. Rep., 2005.

[2] S. Song, A. C. Singer, and K.-M. Sung, "Soft input channel estimation for turbo equalization," IEEE Transactions on Signal Processing, vol. 52, no. 10 , pp. 2885-2894, October 2004.

[3] S. Haykin, Kalman Filtering and Neural Networks. Wiley-Interscience, October 2001.

[4] W. Hackbusch, Iterative Solution of Large Sparse Systems of Equations (Applied Mathematical Sciences). New York, NY: Springer, 1993.

[5] J. R. Shewchuk, "An introduction to the conjugate gradient method without the agonizing pain," School of Computer Science, Carnegie Mellon University, Pittsburgh, PA, Tech. Rep., August 1994.

[6] P. N. Swarztrauber, "The methods of cyclic reduction, Fourier analysis and the FACR algorithm for the discrete solution of Poisson's equation on a rectangle," SIAM Review, vol. 19, no. 3, pp. 490-501, Jul. 1977.

[7] T. J. Riedl and A. C. Singer, "Iterative state estimation," Tech. Rep., 2010, draft.

[8] K. E. Baddour and N. C. Beaulieu, "Autoregressive models for fading channel simulation," Proc. IEEE Global Telecommunications Conference, November 2001.

[9] P. Bello, "Characterization of randomly time-variant linear channels," IEEE Transactions on Communication Systems, vol. 11, no. 4, pp. 360393, Dec. 1963. 